\title{
Turbulent Dynamics of Epithelial Cell Cultures
}

\author{
C. Blanch-Mercader, ${ }^{1, *}$ V. Yashunsky, ${ }^{1, \dagger}$ S. Garcia, ${ }^{1}$ G. Duclos,${ }^{1}$ L. Giomi ${ }^{2}$ and P. Silberzan ${ }^{1}$ \\ ${ }^{1}$ Laboratoire PhysicoChimie Curie, Institut Curie, PSL Research University-Sorbonne Université, \\ UPMC-CNRS-Equipe labellisée Ligue Contre le Cancer, 75005 Paris, France \\ ${ }^{2}$ Instituut-Lorentz, Universiteit Leiden, P.O. Box 9506, 2300 RA Leiden, The Netherlands
}

(Received 5 November 2017; revised manuscript received 12 February 2018; published 17 May 2018)

\begin{abstract}
We investigate the large length and long time scales collective flows and structural rearrangements within in vitro human bronchial epithelial cell (HBEC) cultures. Activity-driven collective flows result in ensembles of vortices randomly positioned in space. By analyzing a large population of vortices, we show that their area follows an exponential law with a constant mean value and their rotational frequency is size independent, both being characteristic features of the chaotic dynamics of active nematic suspensions. Indeed, we find that HBECs self-organize in nematic domains of several cell lengths. Nematic defects are found at the interface between domains with a total number that remains constant due to the dynamical balance of nucleation and annihilation events. The mean velocity fields in the vicinity of defects are well described by a hydrodynamic theory of extensile active nematics.
\end{abstract}

DOI: 10.1103/PhysRevLett.120.208101

The collective dynamics of cellular systems has gathered increasing attention over the last decades [1], inspiring experimental, theoretical, and numerical studies in an attempt to unravel the underlying physical principles. Single cells are able to convert chemical energy into mechanical forces [2], allowing them to move and modify their local microenvironment [3]. At the multicellular level, these forces are transmitted over tens of cell lengths [4], driving nonlocal cellular flows [5], which self-organizes into a vast range of patterns [6-13]. The large-scale behavior of highly active epithelial monolayers often features signatures of erratic motion [11,12]; however, whether these can be attributed to the intrinsic cellular stochasticity or to some form of collective modes in a turbulent regime remains an open question. Other biological systems like bacterial colonies or suspensions of cytoskeletons' filaments exhibit chaotic dynamics [14-17]. Despite differing in length, time, or energy scales, these systems are often qualitatively described by continuum models [5]. Turbulence in active matter has been reported in Toner-Tu models $[15,18]$, generalized Swift-Hohenberg models [19], and active nematic [20-23] and polar [24,25] gels. In particular, recent theoretical findings have established the characteristic features associated with active nematic turbulence [21], namely, the spatial organization of the turbulent flows is determined by an intrinsic length scale, the area of vortices defined by the collective flows follows an exponential law, and the rotational frequency of vortices is independent of their size. However, these theoretical developments have not yet been accompanied by experimental studies in cellular systems.

In the present Letter, we report the existence of a turbulent regime in human bronchial epithelial cells (HBECs) over three decades in kinetic energy, and we show that our findings are in agreement with an hydrodynamical model of an active nematic fluid.

We study experimentally the nematodynamics of immortalized HBEC monolayers and its time evolution. Contrary to other epithelial cell lines, HBECs are weakly cohesive at the initial stages, meaning that when cells fully cover the substrate, they are still highly motile and exhibit long-range collective movements $[26,27]$. The system gradually slows down approaching asymptotically a jammed state after $\sim 60 \mathrm{~h}$, in which cells hardly move beyond their own sizes [26].

At the intermediate time regimes $(\sim 20 \mathrm{~h})$, HBECs form a cohesive monolayer, showing large-scale collective movements [Fig. 1(a)]. Mapping the velocity field $\left(v_{x}, v_{y}\right)$ [28], we observe large and transient vortices, which show no apparent sign of spatial organization [Fig. 1(b)]. To quantify the global properties of cell flows, we define the in-plane enstrophy $\Omega=\left\langle\omega^{2} / 2\right\rangle$ as the square of the $2 D$ vorticity field $\omega=\partial_{x} v_{y}-\partial_{y} v_{x}$ and the in-plane kinetic energy per unit mass $\mathcal{E}=\left\langle\left(v_{x}^{2}+v_{y}^{2}\right) / 2\right\rangle$, where the symbol $\langle\cdot\rangle$ denotes spatial averages. The squared root of $\mathcal{E} / \Omega$ has units of length, which is roughly half of the mean vortex radius $\ell_{a}$ [15]. The vortex area is quantified using the Okubo-Weiss criterion [29,30], which identifies the elliptic regions around vortices for which the field $Q=-\operatorname{det} \nabla \mathbf{v}<0$ as shown in Fig. 1(d). For incompressible flows, this implies that the velocity gradient tensor has a pair of purely imaginary conjugate eigenvalues [29,30].

Single HBECs are round shaped, but when crowded, they deform and acquire elongated shapes (see Supplemental Material Figs. S1-S3 in [31]). The anisotropy of the shapes allows us to determine a local nematic orientation described 

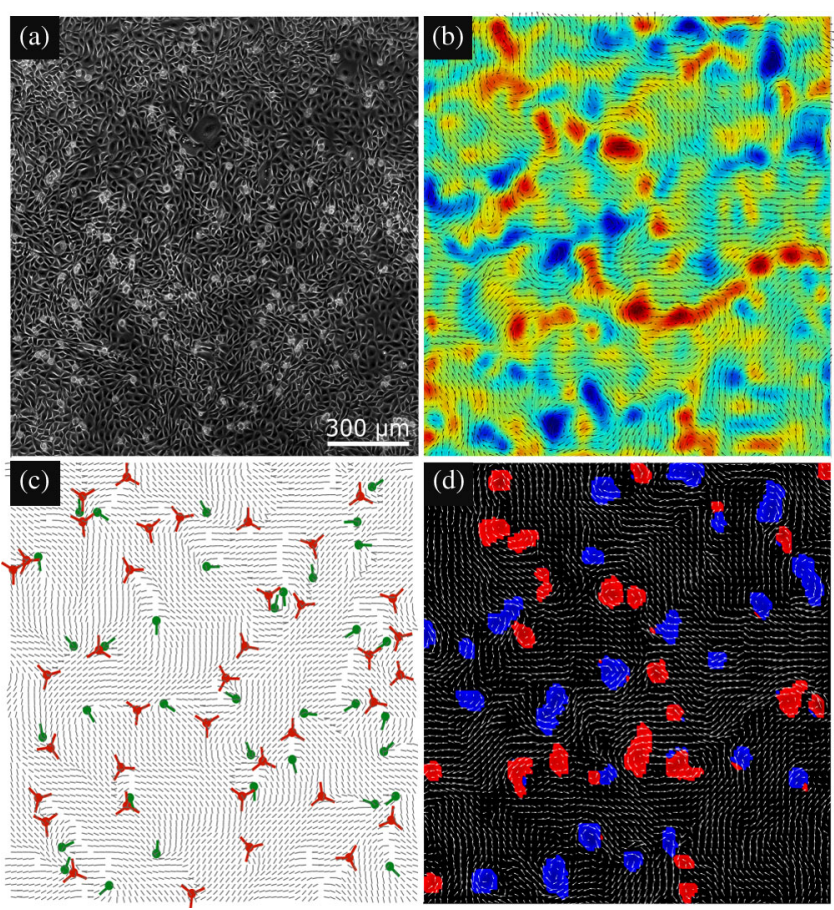

FIG. 1. Nematodynamics of HBEC monolayers. (a) Phase contrast image. (b) Normalized vorticity map with the particle image velocimetry flow field illustrating the collective behavior. (c) Cell orientation map with green (respectively, red) dots marking the position of $+1 / 2$ (respectively, -1/2) defects. (d) Map of the Okubo-Weiss field thresholded to negative values (i.e., $Q<0$ in the text) where blue (respectively, red) domains indicate clockwise (respectively, counterclockwise) rotation. Scale bar is $300 \mu \mathrm{m}$.

by the unit vector $\left(\hat{n}_{x}, \hat{n}_{y}\right)$ (Fig. S1 in [31]). Cells selforganize into transient mesoscopic nematic domains, separated by pairs of $\pm 1 / 2$ defects [Fig. 1(c)]. The position and orientation of $+1 / 2$ (respectively, $-1 / 2$ ) defects are labeled by a green (respectively, red) symbol in Fig. 1(c) [32]. The cell shape anisotropy may be related to the cellular forcegeneration machinery [35].

Over the course of the experiment, cells proliferate, increasing their surface density. As a result, the energy and enstrophy exponentially decrease with time over more than three decades [Fig. 2(a)]. The inverse of the squared root of the enstrophy $\Omega^{-1 / 2}$ defines a characteristic timescale for the instantaneous dynamics of the system, allowing us to average over different experiments. From Fig. 2(a), we conclude that $\Omega^{-1 / 2}$ increases from 0.3 to $8 \mathrm{~h}$, while the collective cell dynamics itself slows down with a typical timescale of $\partial_{t} \Omega / \Omega \sim 10 \mathrm{~h}$ [Fig. 2(a)]. Up to the first $40 \mathrm{~h}$, the separation of these two timescales suggests that the material properties of HBECs are changing adiabatically. Unless otherwise stated, we focus our analysis on this time interval.

Based on the organization of HBEC monolayers, we hypothesize that HBECs behave as active nematic liquid
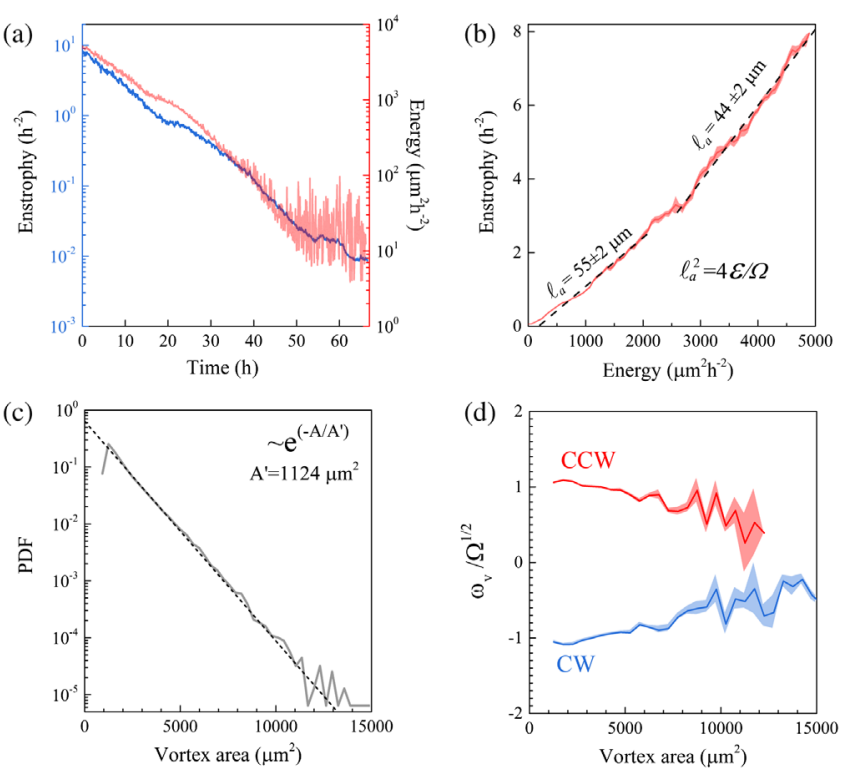

FIG. 2. Statistical analysis of the dynamics of mesoscopic cell flows. (a) Log-linear representation of the time evolution of enstrophy $\Omega$ and kinetic energy per unit mass $\mathcal{E}$. (b) Linear scaling between $\Omega$ and $\mathcal{E}$ over the entire experiment. (c) Loglinear representation of the total vortex area probability density distribution (PDF). The dotted line is an exponential fit. (d) The mean vorticity per vortex normalized by $\Omega^{1 / 2}$ as a function of the vortex area. CW stands for clockwise and $\mathrm{CCW}$ for counterclockwise rotation.

crystals. Theoretical work has showed that the dynamics of active nematics is controlled by a characteristic length scale $\ell_{a}=\sqrt{K /|\alpha|}$, with $K$ as the Frank elastic constant of the nematic phase and $\alpha$ as the active stress, resulting from the balance between active and passive forces [21]. When $\ell_{a}$ is much smaller than the system size, the flow organizes in an ensemble of vortices of average vorticity $\omega_{v} \sim \alpha / \eta$, with $\eta$ as the shear viscosity, and whose area $A$ is exponentially distributed, i.e., $P(A) \sim \exp (-A /\langle A\rangle)$, with $\langle A\rangle \sim \ell_{a}^{2}$. In turn, the nematic director is decomposed in domains of average size $\ell_{a}$ surrounded by $\pm 1 / 2$ disclinations. As a consequence of this underling structure, $\Omega \sim \omega_{v}^{2}$ and $\mathcal{E} \sim \omega_{v}^{2} \ell_{a}^{2}$, thus $\mathcal{E} / \Omega \sim \ell_{a}^{2}[21]$.

Figure 2(b) shows the measured values of energy and enstrophy over the course of the experiment. The ratio $\mathcal{E} / \Omega$ changes only slightly, consistent with a slight increase of the average vortex size $\ell_{a}$ from $44 \pm 2$ to $55 \pm 2 \mu \mathrm{m}$. This may suggest that the time variation of the Frank constant $K$ and the active stress $\alpha$ are very limited. On the other hand, the decay in enstrophy, Fig. 2(a), implies, under the assumption that $\alpha$ remains constant, that the shear viscosity $\eta$ increases by more than a decade. Such an increase in viscosity could be caused by the incipient jamming of the cellular fluid [36-38], as well as by the maturation of cellcell contacts $[26,27]$.

To determine the probability distribution of the vortex area $A$ and their rotational frequency $\omega_{v}$, we proceeded by 
first verifying that their functional form did not change over the course of the experiment (Fig. S4 in [31]). Hence, we analyzed about 10000 independent vortices and found that the vortex area distribution is exponential with a mean value of $1120 \mu \mathrm{m}^{2}$ [Fig. 2(c)], which is on the order of the energy-enstrophy ratio. In addition, we observe a slight decrease of the distribution of the mean vorticity per vortex $\omega_{v} / \Omega^{1 / 2}$ with the vortex area [Fig. 2(d)]. At the accuracy of the experiment, the rotation of vortices is evenly distributed between clockwise and counterclockwise (Fig. S4 in [31]). Altogether, we show that the area of vortices is exponentially distributed and that the mean vorticity per vortex is approximately size independent. The presence of both features together are a clear signature of turbulence in active nematics [17,21].

To further validate the active nematic nature of our cellular cultures, we analyze the statistical properties of cell orientation. HBECs tend to form transient finite-size domains with high orientational order [Fig. 1(c)] [31]. The typical domain size is given by the nematic correlation length $\ell_{c}$ or the decaying length of the director correlation function $\mathcal{C}_{n}=\left\langle\hat{\mathbf{n}}\left(x^{\prime}+x, y^{\prime}+y\right) \cdot \hat{\mathbf{n}}\left(x^{\prime}, y^{\prime}\right)\right\rangle_{\left(x^{\prime}, y^{\prime}\right)}$, where the spatial average is over the entire field of view and $\hat{\mathbf{n}}$ denotes the cell director. $\pm 1 / 2$ defects, which are a hallmark of nematic liquid crystals [39], are found at the interface between these domains. Nematic arrangements were found previously in cultures of fibroblasts, myoblasts, retinal epithelial cells, neural progenitor cells, and kidney epithelial cells [40-43]. In our system, we found that $\ell_{c} \sim$ $100 \mu \mathrm{m}$ is comparable to the mean vortex size, with a slight tendency to increase with time [Fig. 3(a)]. Remarkably, in our system, the total number of defects decreases only slightly from $77 \pm 3$ to $55 \pm 2$ upon a 100-fold variation of the kinetic energy up to the first $40 \mathrm{~h}$ [Fig. 3(a)]. However, unlike in previously described contractile cell monolayers that are dominated by cell-substrate friction and eventually behave as passive liquid crystals [41], in HBEC monolayers, the number of defects is set by the dynamical balance of creation and annihilation events of defect pairs. The rate of these processes, which is on the order $\sim 1 / \mathrm{h}$, decreases by a factor of 2 as tissue begins to jam [Fig. 3(c)]. These confirm altogether that the time variation of $K$ and $\alpha$ are very limited $[20,21]$.

Analyzing the trajectories of individual defects at long timescales (more than $1 \mathrm{~h}$ ), we observe that the $2 \mathrm{D}$ mean squared displacement (MSD) follows a power law with an exponent close to 1, yielding an effective diffusion coefficient of $\sim(34 \pm 2) \times 10^{2} \mu \mathrm{m}^{2} / \mathrm{h}$ with no apparent quantitative differences between both types of defects [Fig. 3(d)]. Note that, in these experiments characterized by a high density of defects, the motion of individual defects is largely controlled by the interactions with the other defects [39]. As a result, the ballistic regime in the mean squared displacement of $+1 / 2$ defects is transient and appears through a short-time superdiffusive behavior
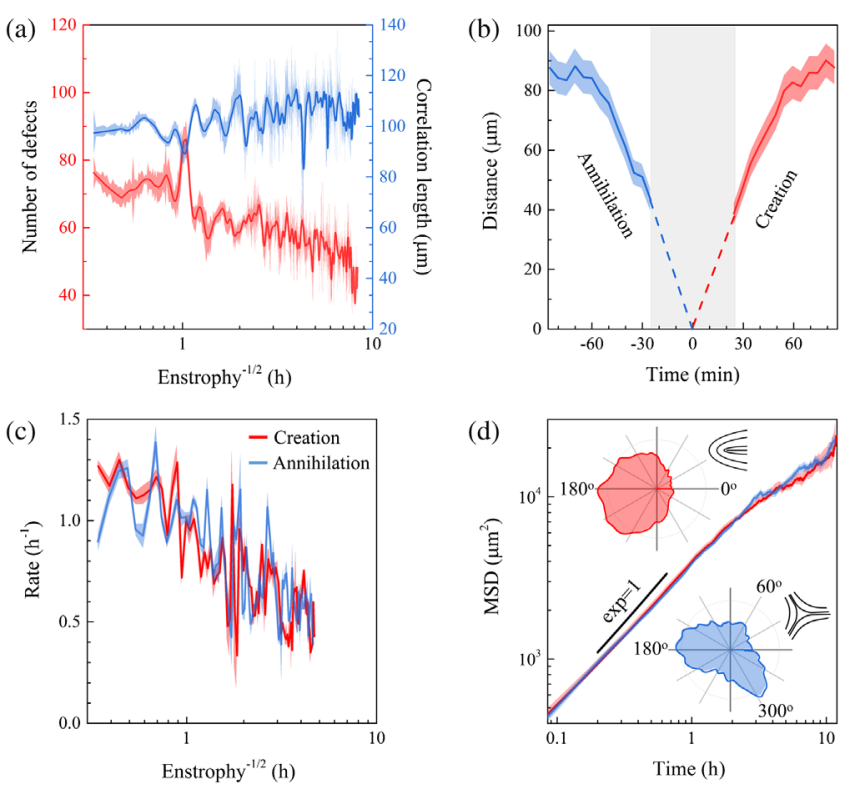

FIG. 3. Statistical analysis of the cell nematic order parameter. (a) Number of defects and nematic correlation length as a function of $\Omega^{-1 / 2}$. We employ the enstrophy to label the time coordinate. (b) Short-time dynamics of the minimal distance between two opposed nematic defects near the nucleation (annihilation) instant in red (blue). (c) Time evolution of the creation and annihilation rates as a function of $\Omega^{-1 / 2}$. (d) Long-time dynamics of MSD of nematic defects. The MSD curves are fitted by $\sim D t^{n}$. (Inset) Distribution of the relative angle between the average velocity at the defect core and the defect orientation.

[21] that we cannot observe here because of the limited accuracy of our experiment. Interestingly, the direction of the instantaneous flow field at the defect core indeed reflects the symmetries of the local extensile nematic arrangement near defects [inset of Fig. 3(d)].

Figure 3(b) shows the trajectories of the minimal distance $\Delta$ between two opposite-charge defects toward an annihilation (blue) or away from a nucleation (red) event, where time is set at the annihilation or creation instant. Their trajectories have opposite but symmetrical profiles [Fig. 3(b)], saturating over distances of $100 \mu \mathrm{m}$, which is comparable to the nematic correlation length $\ell_{c}$. The resolution of our analysis does not allow us to resolve the dynamics closer to the core of the defects. Despite the collective nature of turbulence in active nematics, simple theoretical arguments [44-46] suggest that the distance $\Delta$ varies in time according to $\partial_{t} \Delta= \pm v_{0}-\kappa / \Delta$ (up to higher-order correction in $\Delta$ ), where the positive (respectively, negative) sign applies to the nucleation (respectively, annihilation) case. The coefficient $v_{0}$ stands for the selfpropelled velocity of $+1 / 2$ defects, whereas $\kappa=K / \gamma$, with $\gamma$ as the rotational viscosity of the nematic [44-46]. By fitting this equation on our trajectories from Fig. 3(b), we obtain $v_{0}=1.3 \pm 0.2 \mu \mathrm{m} / \mathrm{min}$ and $\kappa<6 \mu \mathrm{m}^{2} / \mathrm{min}$, suggesting that the nematoelastic interaction between defects is 


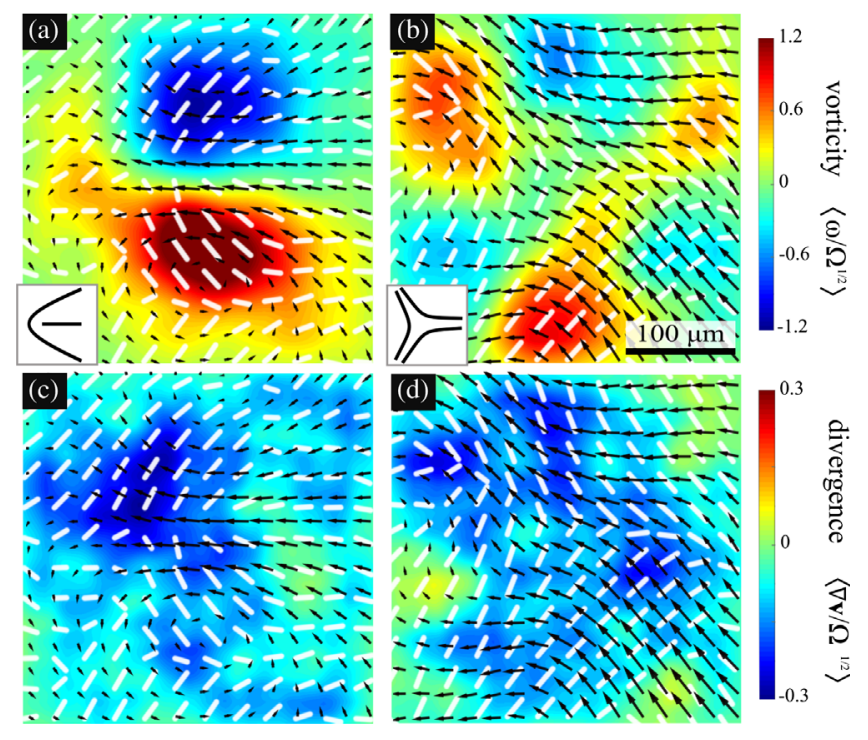

FIG. 4. Ensemble average of the flows near nematic defects. (a) and (c) (respectively, (b) and (d)) show the vorticity and divergence of the velocity field normalized by $\Omega^{1 / 2}$ for a $+1 / 2$ (respectively, $-1 / 2$ ) defect. The overlaid black arrows represent the direction of the mean velocity field. The overlaid white rods represent the mean nematic director. (Upper insets) Sketches of the nematic orientation around the defects.

subdominant against the activity of $+1 / 2$ defects for HBEC monolayers.

To confirm the nematodynamic coupling of the system, we analyze the local properties of cell flows around defects. Here, we compute the average local director and velocity fields for a population of $\sim 10000$ nematic defects [31] (Figs. 4 and S5 [31]). The vorticity map of $+1 / 2$ defects presents a symmetrical organization of a pair of counterrotating vortices [Fig. 4(a)], generating a net flow directed towards the defect head, which is reminiscent of the observations in Madin-Darby canine kidney and neural progenitor cells $[42,43]$. This observation confirms that HBECs behave as extensile nematics. Remarkably, the vorticity map of $-1 / 2$ defects presents a threefold organization of pairs of counterrotating vortices [Fig. 4(b)]. These observations are in excellent agreement with the flow field of isolated defects in extensile active nematics [46]. In addition, weak negative divergence is found near the cores of both type of defects [Figs. 4(c) and 4(d)], which is not captured by incompressible flows (i.e., $\nabla \cdot \mathbf{v}=0$ ). Unlike in neural progenitor cells [43], this observation suggests an accumulation of cells near both types of nematic defects.

In conclusion, we have shown that HBEC monolayers develop $2 D$ turbulent collective flows characterized by the spontaneous emergence of mesoscopic vortices and nematic defects over a wide range of kinetic energy. The properties of cell flows and cell orientation are analyzed for length scales of millimeters and timescales of a few days, granting access to high statistics with which the predicted behavior of active nematics is tested. Our findings show that, regardless of the instantaneous cell density, the statistics of vortices are compatible with the chaotic dynamics of active nematics [21]. In particular, we show that the vortex area distributes exponentially with a time-independent mean value and the vortex rotational frequency is roughly size independent. Furthermore, we analyze the spatiotemporal dynamics of cell orientation and the interplay with the local cell flows. We observe that HBEC monolayers exhibit spontaneous formation and annihilation of pairs of nematic defects of opposite charges, yielding an approximately constant total number of defects. The vorticity of cell flows near $\pm 1 / 2$ defects is in excellent agreement with the flows generated by isolated defects in active liquid crystals $[21,44]$. Therefore, prior to entering into the jamming phase, HBEC monolayers behave as active nematics operating in a regime, where the contractile active stresses $\alpha$ and the Frank constant $K$ are weakly density dependent, and the effective shear viscosity $\eta$ varies more than a decade. We believe that the physical principles governing tissue mechanics can be further explored by analyzing the statistical properties of the spatiotemporal dynamics of cell cultures.

We thank Jacques Prost and Jean-François Joanny for enlightening discussions and Julia Yeomans for suggesting the cell orientation analysis. C. B.-M. thanks Pau Guillamat for suggesting the analysis of the anisotropy level. We gratefully acknowledge the CelTisPhysBio Labex for financial support. V. Y. acknowledges partial funding from the EU PRESTIGE program. L. G. is supported by the Netherlands Organization for Scientific Research (NWO/ $\mathrm{OCW}$ ) via the Frontiers of Nanoscience program and the Vidi scheme. The Biology-Inspired Physics at Mesoscales Group is a part of the CelTisPhysBio Labex and of the Institut Pierre-Gilles de Gennes.

C. B.-M. and V. Y. contributed equally to this work.

* carles.blanch-mercader@curie.fr †ictor.Yashunsky@curie.fr

[1] V. Hakim and P. Silberzan, Rep. Prog. Phys. 80, 076601 (2017).

[2] O. Du Roure, A. Saez, A. Buguin, R. H. Austin, P. Chavrier, P. Siberzan, and B. Ladoux, Proc. Natl. Acad. Sci. U.S.A. 102, 2390 (2005).

[3] P. M. Bendix, G. H. Koenderink, D. Cuvelier, Z. Dogic, B. N. Koeleman, W. M. Brieher, C. M. Field, L. Mahadevan, and D. A. Weitz, Biophys. J. 94, 3126 (2008).

[4] X. Trepat, M. R. Wasserman, T. E. Angelini, E. Millet, D. A. Weitz, J. P. Butler, and J. J. Fredberg, Nat. Phys. 5, 426 (2009).

[5] M. C. Marchetti, J. Joanny, S. Ramaswamy, T. Liverpool, J. Prost, M. Rao, and R. A. Simha, Rev. Mod. Phys. 85, 1143 (2013).

[6] M. Poujade, E. Grasland-Mongrain, A. Hertzog, J. Jouanneau, P. Chavrier, B. Ladoux, A. Buguin, and P. Silberzan, Proc. Natl. Acad. Sci. U.S.A. 104, 15988 (2007). 
[7] T. E. Angelini, E. Hannezo, X. Trepat, M. Marquez, J. J. Fredberg, and D. A. Weitz, Proc. Natl. Acad. Sci. U.S.A. 108, 4714 (2011).

[8] S. R. K. Vedula, M. C. Leong, T. L. Lai, P. Hersen, A. J. Kabla, C. T. Lim, and B. Ladoux, Proc. Natl. Acad. Sci. U.S.A. 109, 12974 (2012).

[9] X. Serra-Picamal, V. Conte, R. Vincent, E. Anon, D. T. Tambe, E. Bazellieres, J. P. Butler, J. J. Fredberg, and X. Trepat, Nat. Phys. 8, 628 (2012).

[10] K. Doxzen, S. R. K. Vedula, M. C. Leong, H. Hirata, N. S. Gov, A. J. Kabla, B. Ladoux, and C. T. Lim, Integr. Biol. 5, 1026 (2013).

[11] M. Deforet, V. Hakim, H. Yevick, G. Duclos, and P. Silberzan, Nat. Commun. 5, 3747 (2014).

[12] S. R. K. Vedula, H. Hirata, M. H. Nai, A. Brugués, Y. Toyama, X. Trepat, C. T. Lim, and B. Ladoux, Nat. Mater. 13, 87 (2014).

[13] G. Duclos, C. Blanch-Mercader, V. Yashunsky, G. Salbreux, J.-F. Joanny, J. Prost, and P. Silberzan, Nat. Phys., DOI: 10.1038/s41567-018-0099-7, 2018.

[14] V. Schaller, C. Weber, C. Semmrich, E. Frey, and A. R. Bausch, Nature (London) 467, 73 (2010).

[15] J. Dunkel, S. Heidenreich, K. Drescher, H. H. Wensink, M. Bär, and R. E. Goldstein, Phys. Rev. Lett. 110, 228102 (2013).

[16] F. C. Keber, E. Loiseau, T. Sanchez, S. J. DeCamp, L. Giomi, M. J. Bowick, M. C. Marchetti, Z. Dogic, and A. R. Bausch, Science 345, 1135 (2014).

[17] P. Guillamat, J. Ignés-Mullol, and F. Sagués, Nat. Commun. 8, 564 (2017).

[18] H. H. Wensink, J. Dunkel, S. Heidenreich, K. Drescher, R. E. Goldstein, H. Löwen, and J. M. Yeomans, Proc. Natl. Acad. Sci. U.S.A. 109, 14308 (2012).

[19] A. U. Oza, S. Heidenreich, and J. Dunkel, Eur. Phys. J. E 39, 97 (2016).

[20] S. P. Thampi, R. Golestanian, and J. M. Yeomans, Phil. Trans. R. Soc. A 372, 20130366 (2014).

[21] L. Giomi, Phys. Rev. X 5, 031003 (2015).

[22] R. Ramaswamy and F. Jülicher, Sci. Rep. 6, 20838 (2016).

[23] M. M. Genkin, A. Sokolov, O. D. Lavrentovich, and I. S. Aranson, Phys. Rev. X 7, 011029 (2017).

[24] P. Marcq, Eur. Phys. J. E 37, 29 (2014).

[25] C. Blanch-Mercader and J. Casademunt, Soft Matter 13, 6913 (2017).
[26] S. Garcia, E. Hannezo, J. Elgeti, J.-F. Joanny, P. Silberzan, and N. S. Gov, Proc. Natl. Acad. Sci. U.S.A. 112, 15314 (2015).

[27] J.-A. Park, J. H. Kim, D. Bi, J. A. Mitchel, N. T. Qazvini, K. Tantisira, C. Y. Park, M. McGill, S.-H. Kim, B. Gweon et al., Nat. Mater. 14, 1040 (2015).

[28] L. Petitjean, M. Reffay, E. Grasland-Mongrain, M. Poujade, B. Ladoux, A. Buguin, and P. Silberzan, Biophys. J. 98, 1790 (2010).

[29] A. Okubo, Deep-Sea Res. Oceanogr. Abstr. 17, 445 (1970).

[30] J. Weiss, Physica (Amsterdam) 48D, 273 (1991).

[31] See Supplemental Material at http://link.aps.org/ supplemental/10.1103/PhysRevLett.120.208101 for details, which includes Refs. [32-34].

[32] A. J. Vromans and L. Giomi, Soft Matter 12, 6490 (2016).

[33] W. Thielicke and E. J. Stamhuis, J. Open Res. Software 2, e30 (2014).

[34] R. Rezakhaniha, A. Agianniotis, J. T. C. Schrauwen, A. Griffa, D. Sage, C. v. Bouten, F. Van de Vosse, M. Unser, and N. Stergiopulos, Biomech. Model. Mechanobiol. 11, 461 (2012).

[35] M. Czajkowski, D. Bi, M. L. Manning, and M. C. Marchetti, arXiv:1710.09405.

[36] S. Henkes, Y. Fily, and M. C. Marchetti, Phys. Rev. E 84, 040301 (2011).

[37] R. Ni, M. A. C. Stuart, and M. Dijkstra, Nat. Commun. 4, 2704 (2013).

[38] L. Berthier, Phys. Rev. Lett. 112, 220602 (2014).

[39] P. De Gennes and J. Prost, Physics of Liquid Crystals (Oxford University Press, New York, 1995).

[40] R. Kemkemer, D. Kling, D. Kaufmann, and H. Gruler, Eur. Phys. J. E 1, 215 (2000).

[41] G. Duclos, C. Erlenkämper, J.-F. Joanny, and P. Silberzan, Nat. Phys. 13, 58 (2017).

[42] T. B. Saw, A. Doostmohammadi, V. Nier, L. Kocgozlu, S. Thampi, Y. Toyama, P. Marcq, C. T. Lim, J. M. Yeomans, and B. Ladoux, Nature (London) 544, 212 (2017).

[43] K. Kawaguchi, R. Kageyama, and M. Sano, Nature (London) 545, 327 (2017).

[44] L. Giomi, M. J. Bowick, X. Ma, and M. C. Marchetti, Phys. Rev. Lett. 110, 228101 (2013).

[45] L. M. Pismen, Phys. Rev. E 88, 050502 (2013).

[46] L. Giomi, M. J. Bowick, P. Mishra, R. Sknepnek, and M. C. Marchetti, Phil. Trans. R. Soc. A 372, 20130365 (2014). 